\title{
Efficacy of the pharmacologic chaperone migalastat in a subset of male patients with the classic phenotype of Fabry disease and migalastat-amenable variants: data from the phase 3 randomized, multicenter, double-blind clinical trial and extension study
}

\author{
Dominique P. Germain, MD, PhD ${ }^{1}{ }^{1}$, Kathy Nicholls, MD², Roberto Giugliani, MD, PhD ${ }^{3}$, \\ Daniel G. Bichet, $\mathrm{MD}^{4}$, Derralynn A. Hughes, MD, PhD ${ }^{5}$, Laura M. Barisoni, $\mathrm{MD}^{6}$, \\ Robert B. Colvin, $\mathrm{MD}^{7}$, J. Charles Jennette, $\mathrm{MD}^{8}$, Nina Skuban, MD ${ }^{9}$, Jeffrey P. Castelli, $\mathrm{PhD}^{9}$, \\ Elfrida Benjamin, $\mathrm{PhD}^{9}$, Jay A. Barth, $\mathrm{MD}^{9}$ and Christopher Viereck, $\mathrm{PhD}^{9}$
}

Purpose: Outcomes in patients with Fabry disease receiving migalastat during the phase 3 FACETS trial (NCT00925301) were evaluated by phenotype.

Methods: Data were evaluated in two subgroups of patients with migalastat-amenable GLA variants: "classic phenotype" ( $n=14$; males with residual peripheral blood mononuclear cell $\alpha$ galactosidase $\mathrm{A}<3 \%$ normal and multiorgan system involvement) and "other patients" $(n=36$; males not meeting classic phenotype criteria and all females). Endpoints included estimated glomerular filtration rate (eGFR), left ventricular mass index (LVMi), Gastrointestinal Symptoms Rating Scale diarrhea subscale (GSRS-D), renal peritubular capillary (PTC) globotriaosylceramide (GL-3) inclusions, and plasma globotriaosylsphingosine (lyso- $\mathrm{Gb}_{3}$ ).

Results: Baseline measures in the classic phenotype patients suggested a more severe phenotype. At month 24, mean (SD) annualized change in eGFR $\mathrm{CKD}_{\mathrm{EPI}}$ with migalastat was -0.3 (3.76)
$\mathrm{mL} / \mathrm{min} / 1.73 \mathrm{~m}^{2}$ in the classic phenotype subgroup; changes in LVMi, GSRS-D, and lyso-Gb 3 were -16.7 (18.64) $\mathrm{g} / \mathrm{m}^{2},-0.9$ (1.66), and -36.8 (35.78) nmol/L, respectively. At month 6, mean PTC GL-3 inclusions decreased with migalastat $(-0.8)$ and increased with placebo (0.3); switching from placebo to migalastat, PTC inclusions decreased by -0.7 . Numerically smaller changes in these endpoints were observed in the other patients.

Conclusion: Migalastat provided clinical benefit to patients with Fabry disease and amenable variants, regardless of disease severity.

Genetics in Medicine (2019) 21:1987-1997; https://doi.org/10.1038/s41436019-0451-z

Keywords: Fabry disease; migalastat; classic; pharmacogenetics; precision medicine

\section{INTRODUCTION}

Fabry disease (OMIM 301500) is a devastating, rare, and progressive X-linked lysosomal disorder caused by pathogenic variants in the GLA gene, resulting in functional deficiency of a-galactosidase A $(\alpha-G a l A) .{ }^{1,2}$ This causes accumulation of globotriaosylceramide (GL-3 or $\mathrm{Gb}_{3}$ ) within lysosomes in various tissues, producing a wide variety of clinical symptoms and manifestations, including peripheral neuropathy, cardiovascular disease, stroke, end-stage renal disease, gastrointestinal disorders, and premature death. ${ }^{1,2}$ Although some GLA variants do not appear to cause disease, ${ }^{1}$ more than 1000 disease-causing variants have been reported, of which $\sim 60 \%$ are missense. ${ }^{3,4}$ In males, disease severity is generally inversely related to residual a-Gal A activity with variants resulting in near or complete loss of function associated with the "classic" early-onset form of the disease. ${ }^{1}$ Females generally have later symptom onset than males, but can develop the classic phenotype due to skewed $\mathrm{X}$ chromosome inactivation pattern.,

\footnotetext{
${ }^{1}$ Division of Medical Genetics and Inserm U1179, University of Versailles, Paris-Saclay University, Montigny, France; ${ }^{2}$ Royal Melbourne Hospital, University of Melbourne, Parkville, VIC, Australia; ${ }^{3}$ Medical Genetics Service, HCPA, and Department of Genetics, UFRGS, Porto Alegre, Brazil; ${ }^{4}$ Department of Nephrology, Hôpital du Sacré-Coeur, University of Montreal, Montreal, QC, Canada; ${ }^{5}$ Royal Free NHS Foundation Trust and University College London, London, UK; ${ }^{6}$ Department of Pathology, University of Miami, Miller School of Medicine, Miami, FL, USA; ${ }^{7}$ Harvard Medical School, Massachusetts General Hospital, Boston, MA, USA; ${ }^{8}$ School of Medicine, University of North Carolina at Chapel Hill, Chapel Hill, NC, USA; ${ }^{9}$ Amicus Therapeutics, Inc, Cranbury, NJ, USA. Correspondence: Dominique P. Germain (dominique.germain@inserm.fr)

Deceased December 19, 2017: Christopher Viereck
} 
Enzyme replacement therapy (ERT) has been the current standard treatment for Fabry disease. ERT has been shown to reduce disease substrate (i.e., GL-3 and plasma globotriaosylsphingosine [lyso- $\left.\mathrm{Gb}_{3}\right]$ ) and symptoms. ${ }^{1,2,7-9}$ However, response to treatment is dependent on several factors including disease severity, and the amount of organ damage present at treatment initiation. ${ }^{10-13}$ Furthermore, lifelong, biweekly intravenous ERT infusions are a burden, which can result in delayed treatment initiation and reduced compliance. Infusion reactions to ERT have been reported, ${ }^{10}$ and development of anti-drug antibodies can reduce the efficacy of ERT, as demonstrated in a 5 -year retrospective analysis in which $40 \%$ of males had serummediated antibody inhibition of agalsidase activity. ${ }^{14}$

Migalastat, a first-in-class, orally administered small molecule, is a pharmacological chaperone that binds to and stabilizes amenable mutant forms of $\alpha$-Gal $\mathrm{A}$, facilitating lysosomal trafficking and increasing lysosomal enzyme activity. $^{4,15-19}$ Amenable mutant forms of $\alpha-G a l$ A are identified using the migalastat amenability assay, which measures migalastat-induced changes in human embryonic kidney (HEK) cells that are transfected with DNA plasmids containing GLA variants. ${ }^{4}$ Established criteria for amenability are an increase in $\alpha$-Gal $A$ activity $\geq 1$.2-fold above baseline and an absolute increase of $\geq 3 \%$ of wild-type (WT) $a$-Gal A after incubation with $10 \mu \mathrm{M}$ migalastat. ${ }^{4}$

In patients with amenable variants, migalastat is a treatment option. In the phase 3, placebo-controlled FACETS study (ClinicalTrials.gov; NCT00925301) in patients with Fabry disease and amenable variants who were either ERT-naive or had not received ERT within the past 6 months, migalastat led to reduced substrates in kidney and plasma, stabilized renal function, reduced cardiac mass, and improved gastrointestinal symptoms. $^{20}$ In the phase 3, active-controlled ATTRACT study (ClinicalTrials.gov; NCT01218659) in ERT-experienced patients, migalastat was associated with a reduction in cardiac mass, had similar effects on renal function compared with ERT, and was generally well-tolerated. ${ }^{19}$ These results led to the approval of migalastat in the European Union, Switzerland, Australia, Republic of Korea, Israel, and Japan for the treatment of Fabry disease in patients over 16 years of age, with amenable GLA variants and GFR $\geq 30 \mathrm{~mL} / \mathrm{min} / 1.73 \mathrm{~m}^{2}$ (refs. ${ }^{15,21}$ ). In addition, migalastat is approved in Canada and the United States for the long-term treatment of Fabry disease in adults ( $\geq 18$ years old) with amenable GLA variants. ${ }^{22,23}$

The objective of the present analyses was to assess the clinical benefit of migalastat in the subset of male patients with the classic phenotype in the FACETS trial (i.e., multiorgan system involvement and residual peripheral blood mononuclear cell [PBMC] $\alpha-\mathrm{Gal} A$ activity $<3 \%$ of normal). ${ }^{20}$ Results in male patients not meeting classic phenotype criteria and all female patients were also assessed.

\section{MATERIALS AND METHODS}

\section{In vitro assays}

Methodology for the Good Laboratory Practice (GLP)validated in vitro assay in HEK-293 cells has been published. ${ }^{4}$
In brief, plasmids containing WT or mutated a-Gal A complementary DNA (cDNA) were used to transfect HEK-293 cells; transfected cells were then incubated in the presence or absence of $10 \mu \mathrm{mol} / \mathrm{L}$ migalastat for 5 days, after which cell lysates were assayed for $\alpha-G a l$ A activity. Variants meeting the prespecified criteria for amenability in the assay were categorized as amenable. ${ }^{4}$

\section{FACETS phase 3 study design}

The multicenter, phase 3, randomized, placebo-controlled, double-blind FACETS trial to evaluate the efficacy and safety of migalastat in patients with Fabry disease and amenable variants has been described in detail. ${ }^{20}$ Briefly, stage 1 of the study consisted of a 6-month, double-blind treatment period in which patients were randomly assigned to receive migalastat $150 \mathrm{mg}$ or placebo every other day. After stage 1, patients could receive open-label migalastat $150 \mathrm{mg}$ every other day for an additional 6 months, and another 12 months during an open-label extension.

The study was approved by the institutional review board or ethics committee at each participating center and was conducted in accordance with the International Conference on Harmonization and Good Clinical Practice guidelines and the principles of the Declaration of Helsinki; all patients provided written informed consent.

\section{Clinical analyses}

Two subgroups of patients with amenable variants enrolled in the FACETS study were identified for evaluation: "classic phenotype" and "other patients." The classic phenotype subgroup included male patients with the classic phenotype of Fabry disease and migalastat-amenable variants; these patients had residual PBMC $\alpha-G a l$ A activity $<3 \%$ normal and multiorgan system involvement, defined as two or more of the following organ system involvements: renal, cardiac, central nervous system, neuropathic pain, and gastrointestinal symptoms. $^{20}$ The other patients subgroup included male patients with migalastat-amenable variants who did not meet the criteria defined above and all females. ${ }^{17,20}$

Changes in measurements of disease severity from baseline are reported. Detailed methodology has been published. ${ }^{20}$ Annualized rates of change from baseline to month 24 in estimated glomerular filtration rate using the Chronic Kidney Disease Epidemiology Collaboration equation (eGFR $\mathrm{CKD}_{\mathrm{EPI}}$ ) and GFR measured using iohexol clearance ( $\left.\mathrm{mGFR}_{\text {iohexol }}\right)$ were calculated based on the slope of the linear regression between the observed values and the assessment times. Mean change from baseline to month 24 in left ventricular mass index (LVMi) was calculated based on $2 \mathrm{D}$ or $\mathrm{M}$-mode echocardiograms assessed by a single, blinded reader at a central laboratory. Changes from baseline in number of GL-3 inclusions per renal peritubular capillary (PTC) were based on kidney biopsies performed at baseline, month 6 , and month 12 , which were assessed per BLISS methodology performed by 3 independent, blinded pathologists. $^{20,24}$ The same three pathologists qualitatively assessed GL-3 changes in podocytes, 
endothelial cells, and mesangial cells. Change from baseline to month 24 in plasma lyso- $\mathrm{Gb}_{3}$ levels was based on measurements of retained iohexol plasma samples from each time point. Twenty-four-hour urine protein levels were measured by a central laboratory. PBMC $\alpha-G a l$ A activity was measured with a fluorescence assay of PBMC lysates and normalized to total protein. ${ }^{4}$

The Gastrointestinal Symptoms Rating Scale was used to assess change from baseline to month 24 in diarrhea symptoms (GSRS-D). A response in the GSRS-D was defined as a reduction $\geq 0.33$. The minimal clinically important difference (MCID) of 0.33 was based on estimates from the literature on several non-Fabry gastrointestinal disorders in which diarrhea is prominent. ${ }^{25,26}$ Distribution-based estimates of MCID in Fabry disease were based on the change from baseline in the placebo arm of the FACETS study, generating an MCID of 0.35 based on half the standard deviation, ${ }^{20}$ supporting an MCID for GSRS-D of 0.33 in Fabry patients.

For all outcomes except PTC GL-3 inclusions, data are pooled for patients within the designated subgroups (classic phenotype and other patients) regardless of treatment allocation for the first 6 months (migalastat or placebo). Change from baseline to month 24 data are the change from baseline to month 24 for patients randomized to migalastat in stage 1 of the study and the change from month 6 (start of migalastat) to month 24 in patients randomized to placebo in stage 1 .

All data were derived from post hoc analyses and formal statistical testing was not performed. Data were analyzed descriptively using mean, standard deviation (SD) or standard error (SE), and 95\% confidence intervals (CIs).

\section{In vitro activity}

\section{RESULTS}

For the GLA variants found in male patients enrolled in this study who met the criteria for the classic phenotype, the mean absolute increase in $\alpha$-Gal A activity with $10 \mu \mathrm{M}$ migalastat in vitro was $24.8 \%$ of WT activity; mean activity increased by 8.2-fold above baseline (Table 1). For all other variants, mean absolute increase in a-Gal A activity in vitro was $24.5 \%$ of WT; mean activity increased by 6.8 -fold above baseline (Table 1).

\section{Clinical data}

\section{Baseline characteristics}

The classic phenotype subgroup includes 14 males with the classic phenotype (16-62 years old). The other patients subgroup includes 36 patients (4 males with nonclassic phenotype [33-58 years old], 32 females [27-68 years old]). Disease severity of patients in the classic phenotype subgroup was evident from baseline measurements of variables and medical history, indicating multisystem involvement (Table 2 and Fig. 1a-e). Mean (SD) baseline eGFR $\mathrm{CKD}_{\text {-EPI }}$ values were 87.8 (33.6) and $95.3(19.6) \mathrm{mL} / \mathrm{min} / 1.73 \mathrm{~m}^{2}$, and mean (SD) baseline $\mathrm{mGFR}_{\text {iohexol }}$ values were 78.6 (22.9) and 88.2 (22.0)
$\mathrm{mL} / \mathrm{min} / 1.73 \mathrm{~m}^{2}$ in the classic phenotype and other patients subgroup, respectively.

GLA variants in male patients with the classic phenotype were p.Ile253Thr $(n=2)$, p.Pro259Arg $(n=2)$, p.Gly183Asp, p.Leu243Phe, p.Cys174Arg, p.Asp55Val/Gln57Leu, p.Gly144Val, p.Arg301Gln, p.Gly373Ser, p.Asp322Glu, p.Gly325Arg, and p.Tyr216Cys ( $n=1$ each).

Six $(43 \%)$ male patients with the classic phenotype were taking angiotensin-converting enzyme inhibitors, angiotensin receptor blockers, or renin inhibitors at baseline; the dosages of these medications had been stable for $\geq 4$ weeks before the screening visit and did not change during the study.

\section{Renal function}

The mean (SD) annualized rate of change in eGFR $\mathrm{CKD}_{\mathrm{EPI}}$ from baseline to month 24 was $-0.3(3.76) \mathrm{mL} / \mathrm{min} / 1.73 \mathrm{~m}^{2}$ (95\% CI: $-2.80,2.25 ; n=11)$ in the classic phenotype subgroup and -0.3 (4.47) $\mathrm{mL} / \mathrm{min} / 1.73 \mathrm{~m}^{2}$ (95\% CI: $\left.-2.0,1.4 ; n=30\right)$ in other patients (Fig. 1a; individual values for male patients with the classic phenotype in Table S1). Median annualized rates of change in the classic phenotype and other patients subgroups were $0.25 \mathrm{~mL} / \mathrm{min} / 1.73 \mathrm{~m}^{2}$ ( $\left.\min , \max :-8.6,4.3\right)$ and 0.33 $\mathrm{mL} / \mathrm{min} / 1.73 \mathrm{~m}^{2}$ ( $\min , \max :-13.8,7.4$ ), respectively. Mean (SD) annualized rate of change in $\mathrm{mGFR}_{\text {iohexol was }-3.0}$ (6.04) $\mathrm{mL} / \mathrm{min} / 1.73 \mathrm{~m}^{2}$ (95\% CI: $-7.65,1.64 ; n=9$ ) in the classic phenotype subgroup and $-1.0(8.66) \mathrm{mL} / \mathrm{min} / 1.73 \mathrm{~m}^{2}$ (95\% CI: $-4.39,2.33 ; n=28)$ in other patients. One male patient with the classic phenotype had a reduction of -16 $\mathrm{mL} / \mathrm{min} / 1.73 \mathrm{~m}^{2}$ from month 6 to month 24. Median (range) change in $\mathrm{mGFR}_{\text {iohexol }}$ from baseline to month 24 was -1.03 $\mathrm{mL} / \mathrm{min} / 1.73 \mathrm{~m}^{2}$ ( $\min , \max :-16.2,3.0$ ) in the classic phenotype subgroup and $-0.34 \mathrm{~mL} / \mathrm{min} / 1.73 \mathrm{~m}^{2}$ (min, max: $-20.4,15.8)$ in the other patients subgroup.

Twenty-four-hour urine protein levels showed small increases from baseline to month 24 in both subgroups. Baseline mean (SD) 24-hour urinary protein excretion values were 767.7 (710.7) (95\% CI: 357.4, 1178.0; $n=14)$ and 360.6 (508.7) $(95 \%$ CI: $183.1,538.1 ; n=34) \mathrm{mg} / 24$ hours in the classic phenotype and other patients subgroups, respectively. Mean (SD) changes from baseline to month 24 in 24-hour urine protein were 271.3 (419.6) (95\% CI: $-79.5,622.0 ; n=8)$ and 168.0 (494.4) (95\% CI: $-20.0,356.1 ; n=29) \mathrm{mg} / 24$ hours in the classic phenotype and other patients subgroups, respectively.

Results were similar to those reported for the overall amenable FACETS population, in which migalastat stabilized eGFR in patients with amenable GLA variants, with mean (SE) annualized changes from baseline to month 24 in $\mathrm{eGFR}_{\mathrm{CKD}-\mathrm{EPI}}$ and $\mathrm{mGFR}_{\text {iohexol }}$ of $-0.3(0.66)$ and $-1.5(1.33)$ $\mathrm{mL} / \mathrm{min} / 1.73 \mathrm{~m}^{2}$, respectively. ${ }^{20}$

\section{Cardiac geometry}

Migalastat led to reductions in LVMi in both subgroups. Of note, mean (SD) baseline LVMi values were $114.3(27.3) \mathrm{g} / \mathrm{m}^{2}$ in male patients with the classic phenotype $(n=14)$ and 88.2 (32.3) $\mathrm{g} / \mathrm{m}^{2}$ in other patients $(n=30)$. Seven $(50 \%)$ males with the classic phenotype and $4(11 \%)$ in the other patients 


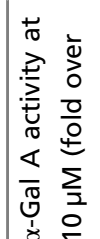
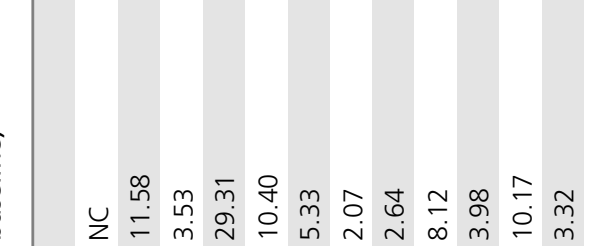

$+\frac{10}{0}$

苛苛

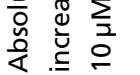

○

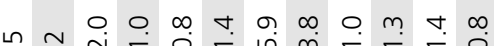

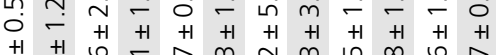

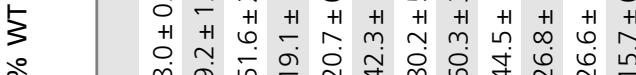

年

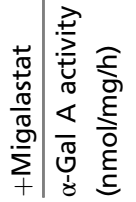

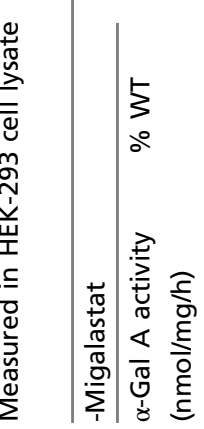

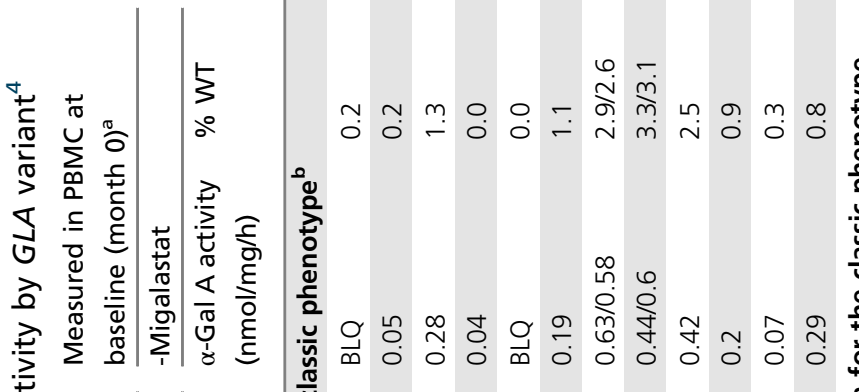

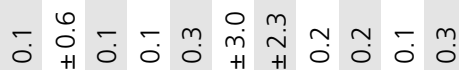

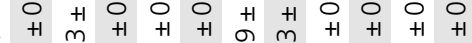

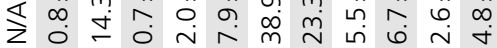

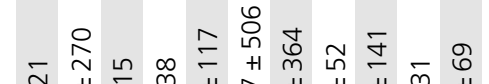

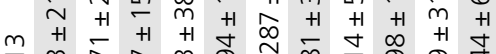

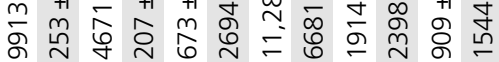

。

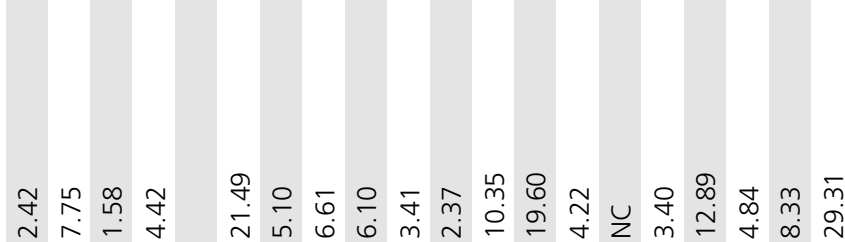

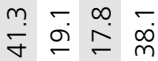

の 6 ․

$\nabla 0 \wedge 6$

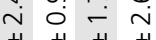

$\begin{array}{llll}0 & 0 \\ 0 & 0 & 0 & 0 \\ +1 & +1 & +1 & 1\end{array}$

হं

vo

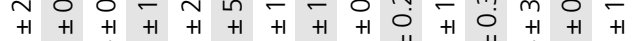

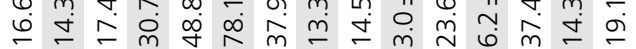

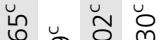

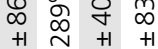

$m+\frac{1}{m} \bar{n}$

过

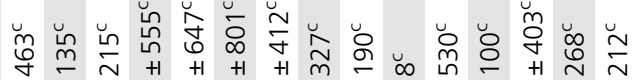

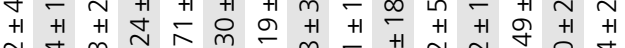

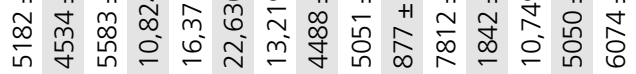

$\stackrel{\infty}{-}-\underset{i}{-} \hat{i}$

- -

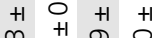

㝏

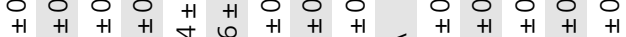

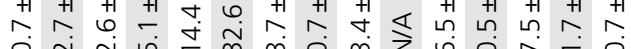

- $\stackrel{\infty}{m}$ o

S $\bar{m}+\frac{m}{n}$

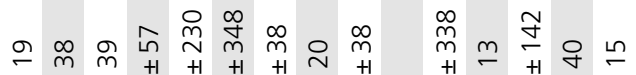

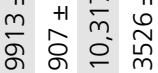

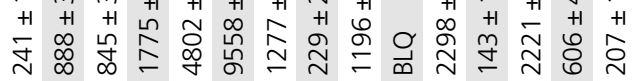

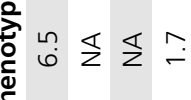

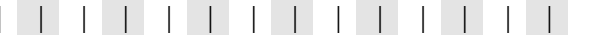

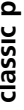

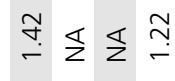

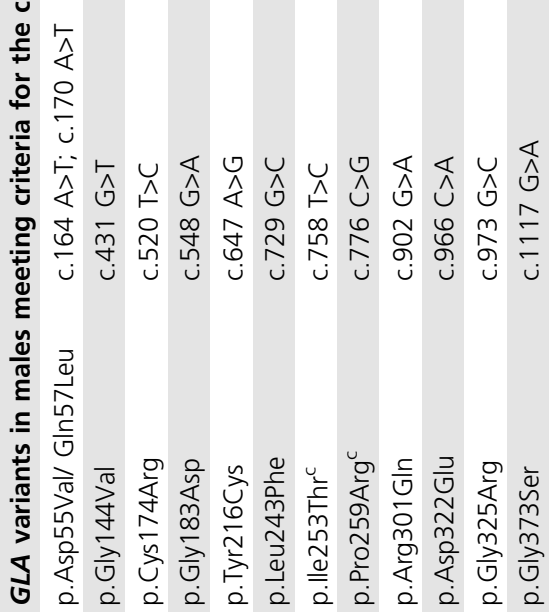

है 


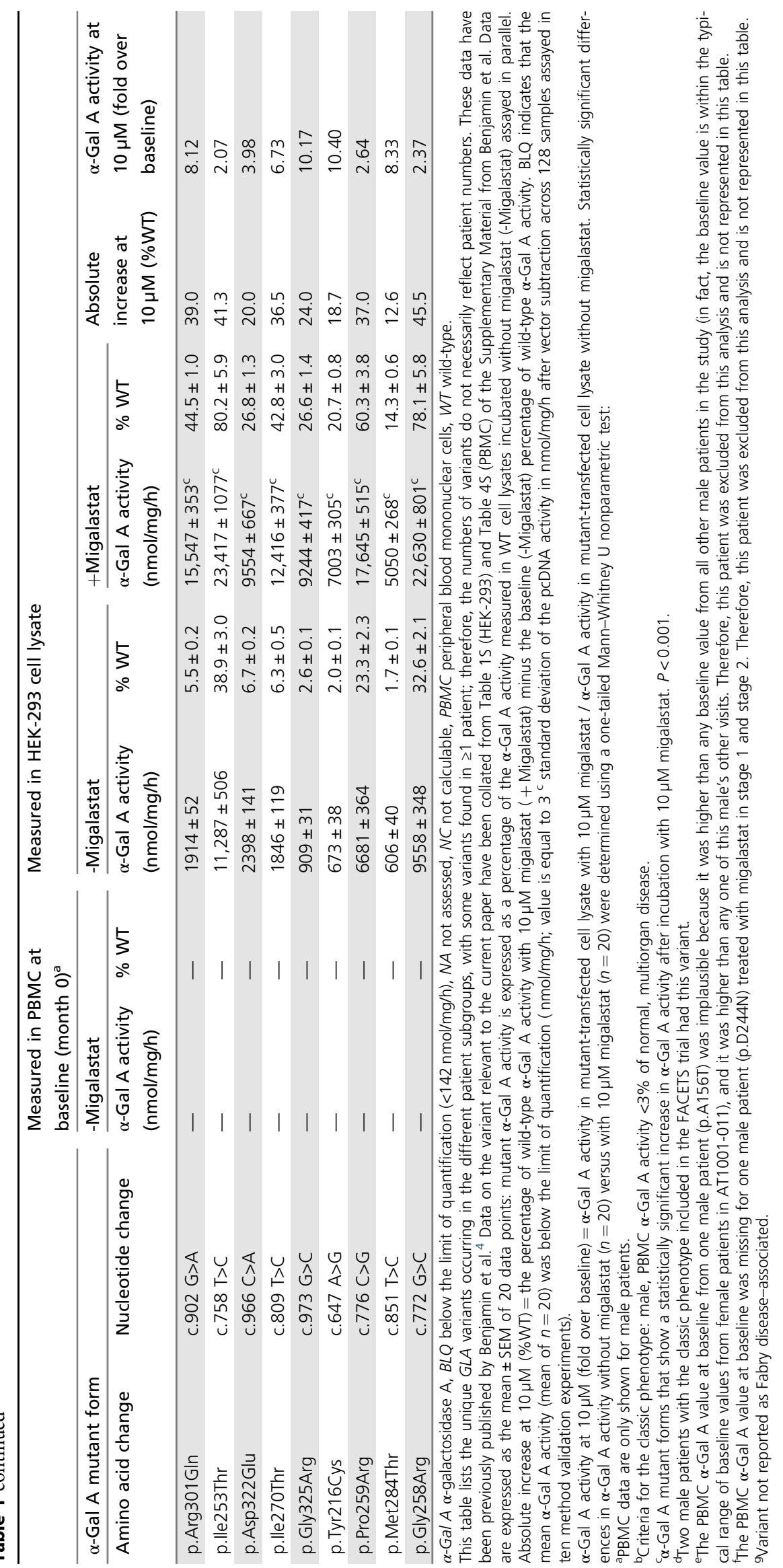




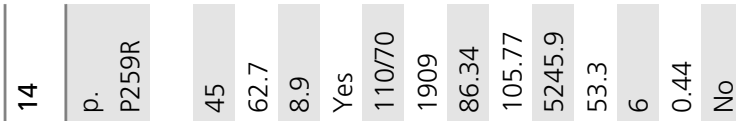

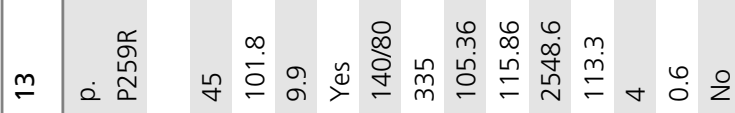

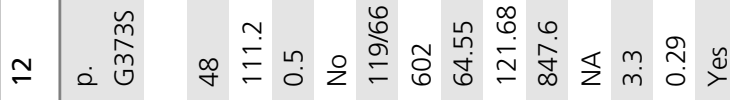

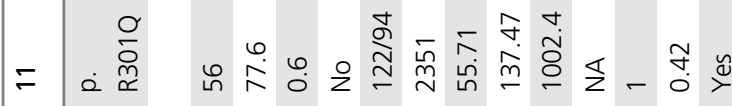



$\circ \stackrel{0}{0}$

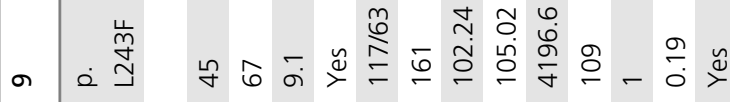

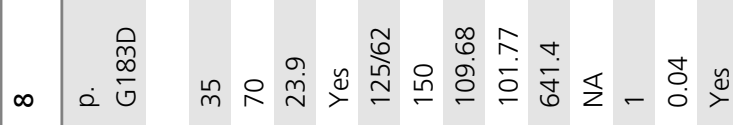

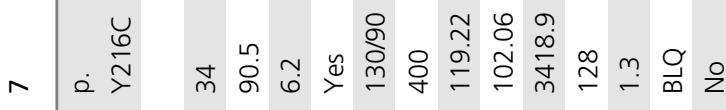

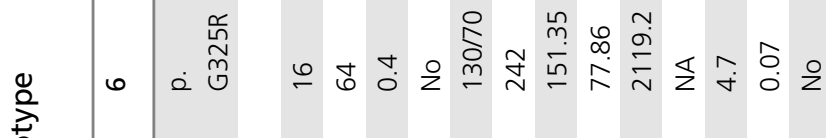

窎

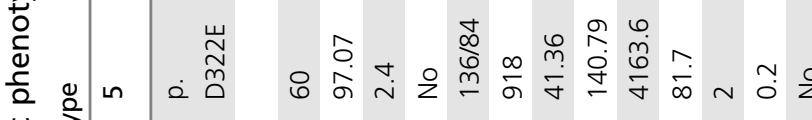

政

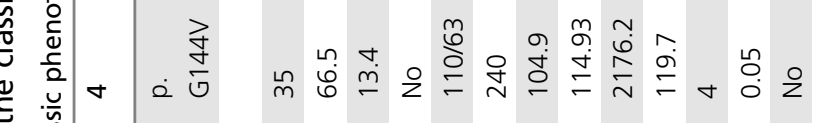

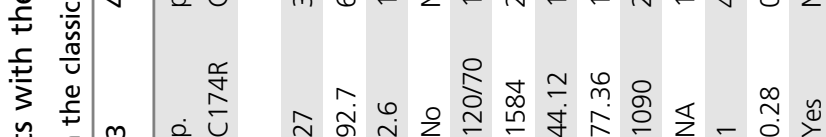

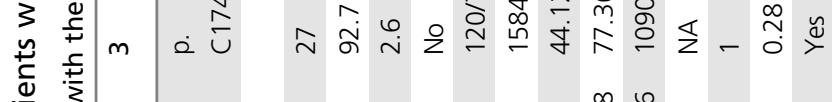

跡

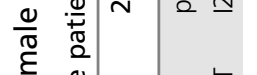

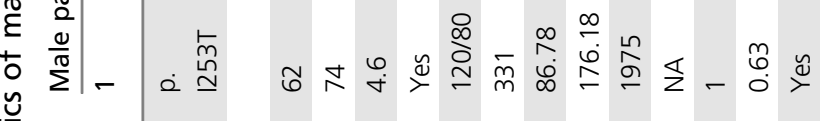

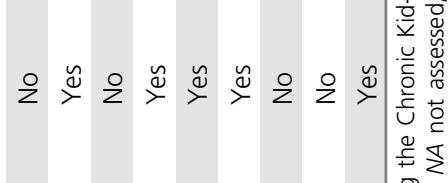

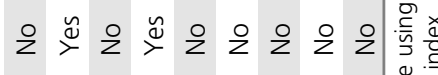

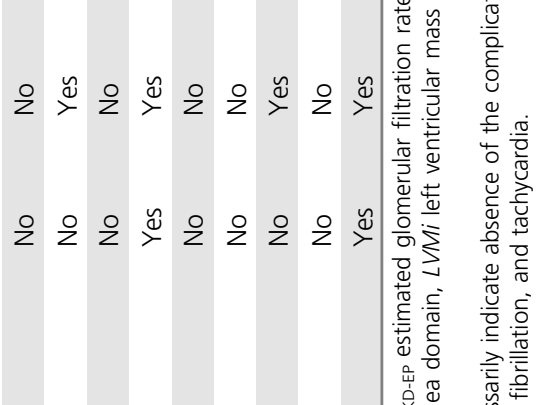

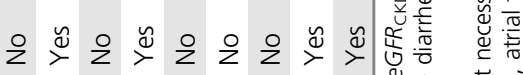

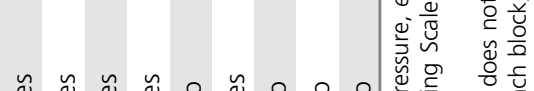

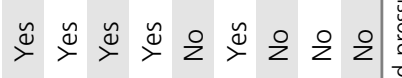

은 듄

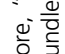

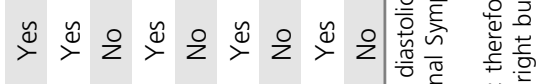

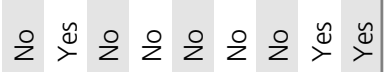

훈

인

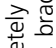

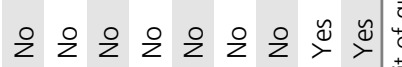

票众

舟.

d.

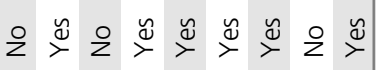

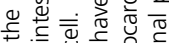

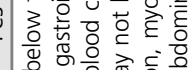

ब记

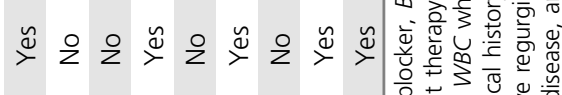

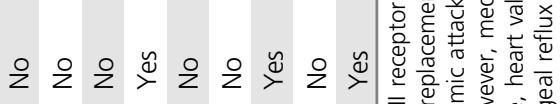

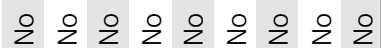

들 흔

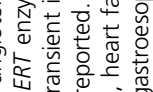

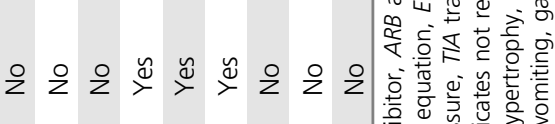

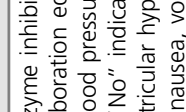

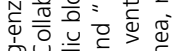

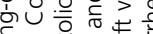

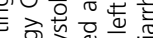

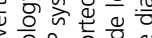

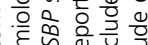

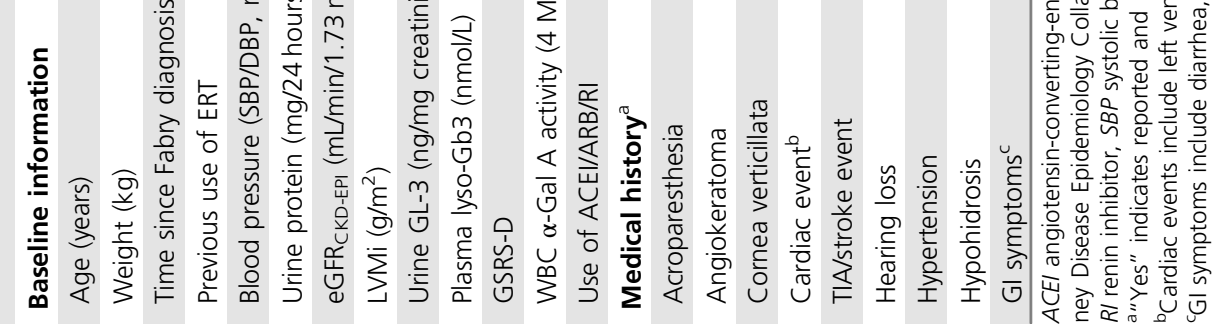




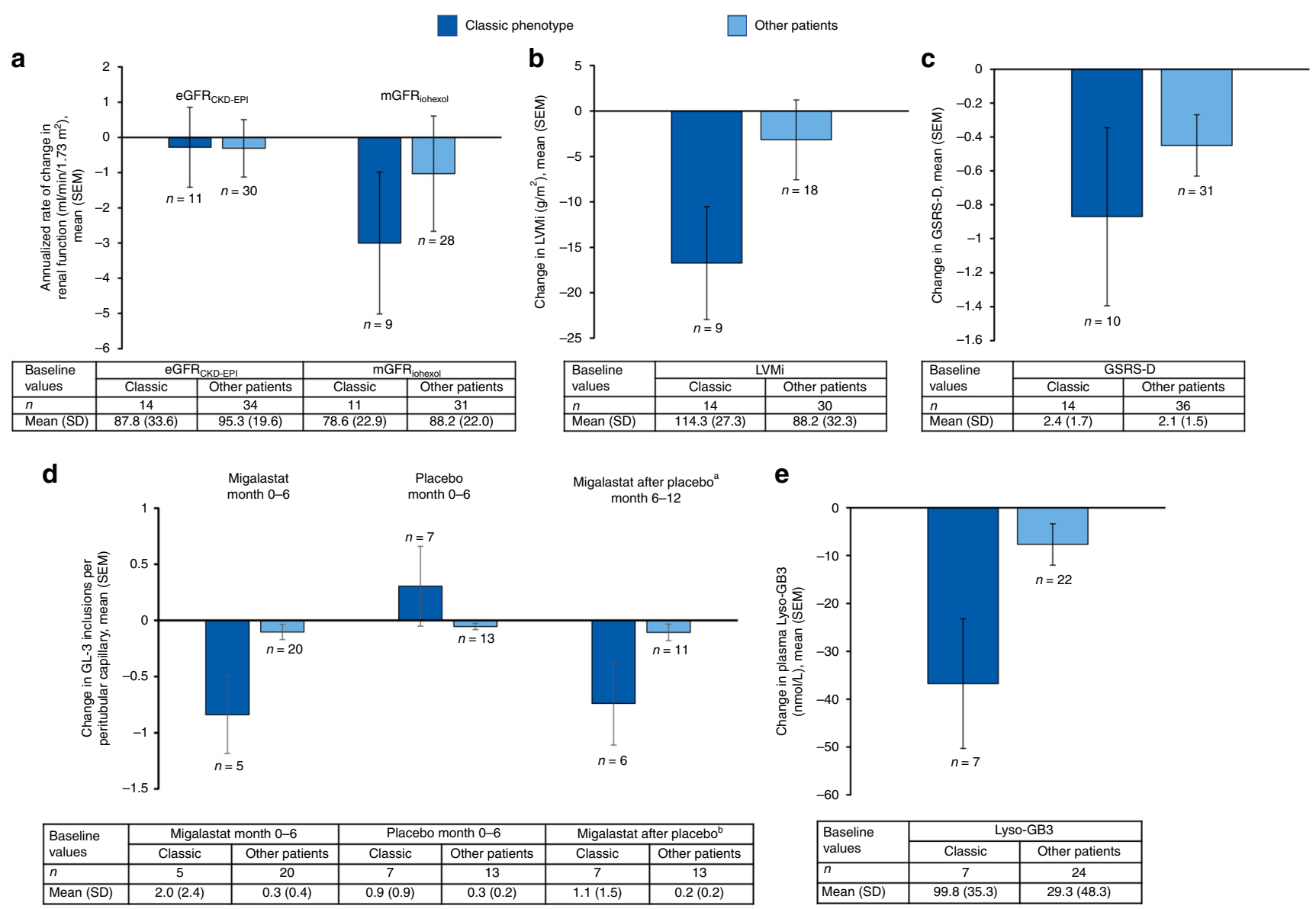

Fig. 1 Measurements of disease severity and treatment effect in the classic phenotype and other patient subgroups. (a) Mean annualized rate of change in estimated glomerular filtration rate (eGFR) and measured glomerular filtration rate (mGFR) from baseline to month 24. (b) Mean change in LVMi from baseline to month 24. (c) Mean change in GSRS-D from baseline to month 24. (d) Mean change from baseline to month 12 in average number of GL-3 inclusions per peritubular capillary. Within each subgroup, patients are grouped according to treatment allocation (migalastat to migalastat or placebo to

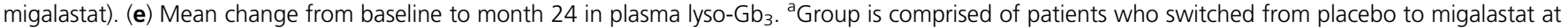
month 6 . ${ }^{b}$ Data are mean (SD) number of GL-3 inclusions per peritubular capillary at month 6 (i.e., after 6 months of placebo treatment). The upper cutoff value for normal plasma lyso-Gb3 level is $2.4 \mathrm{nmol} / \mathrm{L}^{38}$ eGFR $C K D$-EPI estimated glomerular filtration rate using the Chronic Kidney Disease Epidemiology Collaboration equation, GL-3 globotriaosylceramide, GSRS-D Gastrointestinal Symptoms Rating Scale diarrhea subscale, LVMi left ventricular mass index, lyso-Gb 3 plasma globotriaosylsphingosine, $m G F R_{\text {iohexol }}$ glomerular filtration rate measured using iohexol clearance.

subgroup had left ventricular hypertrophy (LVH; $>115 \mathrm{~g} / \mathrm{m}^{2}$ for males; $>95 \mathrm{~g} / \mathrm{m}^{2}$ for females) at baseline. Mean (SD) change from baseline to month 24 in LVMi was - 16.7 (18.64) $\mathrm{g} / \mathrm{m}^{2}$ (95\% CI: $\left.-31.1,-2.4 ; n=9\right)$ in males with the classic phenotype and $-3.2(18.66) \mathrm{g} / \mathrm{m}^{2}(95 \%$ CI: $-12.5,6.1 ; n=$ 18) in other patients (Fig. 1b; individual values for male patients with the classic phenotype in Table S1).

By contrast, LVMi change in the overall migalastat-treated amenable population in FACETS was $-7.7 \mathrm{~g} / \mathrm{m}^{2}$ (95\% CI: $-15.4,-0.01 ; n=27)$ after $18-24$ months of treatment with migalastat, with larger decreases in the subgroup of patients with LVH $\left(-18.6 \mathrm{~g} / \mathrm{m}^{2}\right.$ [95\% CI: $\left.\left.-38.2,1.0\right] ;-11.5 \% ; n=8\right)$ $\left(\right.$ ref. ${ }^{20}$ ).

\section{Gastrointestinal signs and symptoms}

Mean (SD) GSRS-D scores at baseline were $2.4(1.69 ; n=14)$ in the classic phenotype subgroup and $2.1(1.49 ; n=36)$ in the other patients subgroup. Eight (57\%) male patients with the classic phenotype had diarrhea symptoms at baseline (GSRS-D score $\geq 1$ ). In the classic phenotype subgroup, 6 months of treatment (stage 1) with migalastat improved (reduced) scores in GSRS-D (mean [SD] change from baseline -0.3 [0.77]; 95\% CI: $-1.0,0.4 ; n=7$ ), whereas patients treated with placebo had a small increase $(0.2$ [0.46]; $95 \%$ CI: -0.2 , $0.7 ; n=7)$.

Male patients with the classic phenotype benefited from continuing treatment with migalastat or by switching from placebo to migalastat. By 24 months, the mean (SD) change from baseline in diarrhea symptoms was $-0.9(1.66 ; n=10)$ (Fig. 1c; individual values in Table S1). Seven of the 8 (88\%) males with the classic phenotype that had diarrhea symptoms at baseline achieved an MCID reduction of 0.33 in GSRS-D. The corresponding change in other patients was $-0.5(1.01 ; n$ $=31$ ) at month 24 .

These results are similar to those of the overall amenable FACETS population, in which patients with amenable GLA 
variants treated with migalastat had mean changes in GSRS-D of -0.3 (versus 0.2 in the placebo group) at month 6 and -0.5 (95\% CI: $-0.9,-0.1)$ at month 24 (ref. ${ }^{20}$ ).

\section{PTC GL-3 inclusions}

During the first 6 months of the study, the mean (SD) number of GL-3 inclusions per PTC decreased from baseline in males with the classic phenotype treated with migalastat $(-0.8$ [-0.78]; 95\% CI: $-1.8,0.1 ; n=5$ ) (Fig. 1d; individual values in Table S1). Conversely, PTC GL-3 inclusions increased (mean [SD], 0.3 [0.94]; 95\% CI: $-0.6,1.2 ; n=7$ ) during that period in those receiving placebo. Upon switching from placebo to migalastat at month 6, PTC GL-3 inclusions decreased by a mean (SD) of $-0.7(0.91)(95 \% \mathrm{CI}:-1.7,0.2 ; n=6)$ over the next 6 months in these patients. These results mirror those reported for the overall amenable FACETS population, in which PTC GL-3 inclusions decreased over 6 months of migalastat treatment (mean $[\mathrm{SE}],-0.25[0.10] ; n=25$ ) and increased (mean [SE], 0.07 [0.13]; $n=20$ ) over 6 months of placebo treatment. ${ }^{20}$ In the overall amenable FACETS population, a subsequent mean (SE) decrease in mean GL-3 inclusions per PTC of $-0.33(0.15 ; n=17)$ was seen following switch from placebo to migalastat at month 6 .

Decreases in mean (SD) number of PTC GL-3 inclusions were seen in other patients whether treated with migalastat from month 0 to $6(-0.1$ [0.30]; $95 \%$ CI: $-0.2,0.0 ; n=20)$ or switched from placebo to migalastat at month 6 (change from month 6 to $12 ;-0.1$ [0.24]; 95\% CI: $-0.3,0.1 ; n=11$ ) (Fig. 1d). A small decrease was also noted in other patients treated with placebo from month 0 to 6 ( -0.05 [0.10]; 95\% CI: $-0.1,0.0 ; n=13)$. Overall, the decreases in the other patients subgroup were small, likely due to low baseline levels; however, the magnitude of change with migalastat was twice that of placebo.

\section{GL-3 in glomerular cells}

On the basis of qualitative assessments of kidney biopsy samples, patients in the classic phenotype subgroup had either a reduction or no change in GL-3 inclusions in glomerular endothelial cells (3/9 decreased, $6 / 9$ had no change), mesangial cells (3/9 decreased, 6/9 had no change), and podocytes (9/9 had no change) at month 12 compared with baseline or month 6 (for patients switched from placebo to migalastat). No patient showed increase in GL-3 in these cell types after 6-12 months of migalastat treatment (Table S2).

A substantial portion of patients in the other patients subgroup had a reduction in GL-3 in glomerular cells, including mesangial cells (14/31), endothelial cells (8/31), and podocytes (6/31) at month 12 . One patient had an increase in GL-3 in mesangial cells. The remaining patients experienced no change in GL-3 inclusions in these cells after 6-12 months of migalastat treatment.

\section{Plasma lyso- $\mathrm{Gb}_{3}$}

Migalastat was associated with a reduction in plasma lyso- $\mathrm{Gb}_{3}$ in both subgroups, with a considerable reduction observed in the classic phenotype subgroup. The average (SD) change from baseline to month 24 in plasma lyso- $\mathrm{Gb}_{3}$ was -36.8 (35.78) nmol/L (95\% CI: $-69.9,-3.7 ; n=7$ ) for males with the classic phenotype and $-7.66(20.27) \mathrm{nmol} / \mathrm{L}$ (95\% CI: $-16.6,1.3 ; n=22$ ) for other patients (Fig. 1e; individual values in Table S1). Notably, mean (SD) baseline lyso- $\mathrm{Gb}_{3}$ levels were $99.8(35.28 ; n=7) \mathrm{nmol} / \mathrm{L}$ in the classic phenotype subgroup versus $29.3(48.32 ; n=24) \mathrm{nmol} / \mathrm{L}$ in the other patients subgroup.

\section{PBMC $\alpha$-Gal $A$ activity}

Within the classic phenotype subgroup, 6 months of randomized treatment with migalastat led to a mean (SD) increase in PBMC a-Gal A activity of 2.2 (3.0) $4 \mathrm{MU} / \mathrm{h} / \mathrm{mg}$ (95\% CI: $-0.5,5.0 ; n=7)$, whereas treatment with placebo led to a substantially smaller increase $(0.1[0.1] 4 \mathrm{MU} / \mathrm{h} / \mathrm{mg}$; 95\% CI: $0.0,0.2 ; n=7)$. Increase in PBMC $\alpha$-Gal A activity was sustained during open-label migalastat; mean (SD) change from baseline to month 24 was 2.6 (2.3) $4 \mathrm{MU} / \mathrm{h} /$ mg (95\% CI: 0.8, 4.4; $n=9$ ). Changes in PBMC a-Gal A activity appeared to be more variable in the other patients subgroup due to the small number of male patients; mean (SD) change from baseline to month 24 was 2.7 (9.8) $4 \mathrm{MU} / \mathrm{h} / \mathrm{mg}$ (95\% CI:-21.7, 27.1; $n=3$ ).

\section{Adverse events}

The most frequently reported adverse event that occurred during migalastat treatment was headache in both the classic phenotype (4/14 [29\%]) and other patients (15/36 [42\%]) subgroups. The other patients subgroup did not report any other adverse events that occurred in $\geq 20 \%$ of patients, whereas diarrhea, procedure pain, and vertigo were reported in $\geq 20 \%$ of patients in the classic phenotype subgroup (Table S3).

\section{DISCUSSION}

Fabry disease is associated with a wide spectrum of disease progression and clinical phenotypes, ranging from the most severe, classic phenotype to an asymptomatic phenotype in a subset of female patients. ${ }^{1}$ Newborn screening studies estimate the frequency of the classic phenotype as up to 1 in 22,570 males. $^{27}$ Patients with the classic phenotype typically have onset of symptoms during childhood or adolescence, followed by multiorgan complications that can progress to organ failure. ${ }^{1}$ Therefore, early treatment initiation is crucial in this patient population. Current treatment guidelines and consensus recommendations recommend ERT treatment for all males $\geq 16$ years old with classic Fabry disease, even if they are asymptomatic, presenting a high treatment burden. ${ }^{28}$

In a previously published report from the phase 3 FACETS study, patients with Fabry disease who have amenable variants were shown to benefit from treatment with migalastat. $^{20}$ The results presented here provide additional evidence for the beneficial effects of migalastat in male patients with the classic phenotype-the most severe form of Fabry disease. 
In vitro assay data suggest that all patients with Fabry disease and amenable variants, including those with the classic phenotype, would benefit from migalastat treatment, as migalastat increases $\alpha$-Gal A activity in the lysates of cells expressing mutated enzyme. ${ }^{4}$ This hypothesis is supported by our data, which demonstrate reduction in cardiac mass, improvements in GSRS-D, stabilization of eGFR $\mathrm{CKD}_{\text {-EPI }}$, reduction in PTC GL-3 inclusions, plasma lyso- $\mathrm{Gb}_{3}$, and PBMC $a-G a l$ A activity in male patients with the classic phenotype treated with migalastat for up to 24 months.

Compared with published data for male patients with Fabry disease, the results of this analysis suggest that patients treated with migalastat had better renal ${ }^{29-32}$ and $\operatorname{cardiac}^{33,34}$ outcomes than that historically reported for untreated patients. Historical studies of untreated males report an annualized change in eGFR of up to $-12.2 \mathrm{~mL} / \mathrm{min} / 1.73 \mathrm{~m}^{2} .^{29-32}$ Comparatively, the annualized change in eGFR $\mathrm{CKD}_{\mathrm{EPI}}$ was $-0.3 \mathrm{~mL} / \mathrm{min} / 1.73 \mathrm{~m}^{2}$ in the subgroup of patients with the classic phenotype treated with migalastat in FACETS (Fig. 2a). For LVMi, studies of untreated males report annualized increases ranging from 4.1 to $8.0 \mathrm{~g} / \mathrm{m}^{2}$ (refs. ${ }^{33,34}$ ), compared with an annualized change of $-10.4 \mathrm{~g} / \mathrm{m}^{2}$ with migalastat in males with the classic phenotype (Fig. 2b). Furthermore, migalastat appears to provide potential cardiac benefit compared with ERT (Fig. 2) (refs. ${ }^{12,19,34-36}$ ). This is further supported by the greater efficacy of migalastat versus ERT in reducing cardiac mass at month 18 in a phase 3, open-label, randomized clinical trial (ATTRACT; NCT01218659) (ref. ${ }^{19}$ ). The benefit of migalastat on cardiac structure may reflect the broad tissue distribution of migalastat as an orally administered small molecule, including penetration into cardiac tissue. ${ }^{19,23}$ However, the mechanism by which migalastat reduces LVMi is unknown. Clearance of GL-3 deposits from cardiomyocytes and vascular endothelial cells may have contributed to the mass reduction; GL-3 clearance from the latter could also lead to enhanced regional myocardial perfusion and function, influencing the dynamic of left ventricular remodeling. Additionally, it appeared that reduction in LVMi was due to a reduction in both wall thickness and cavity size (Amicus Therapeutic data on file).
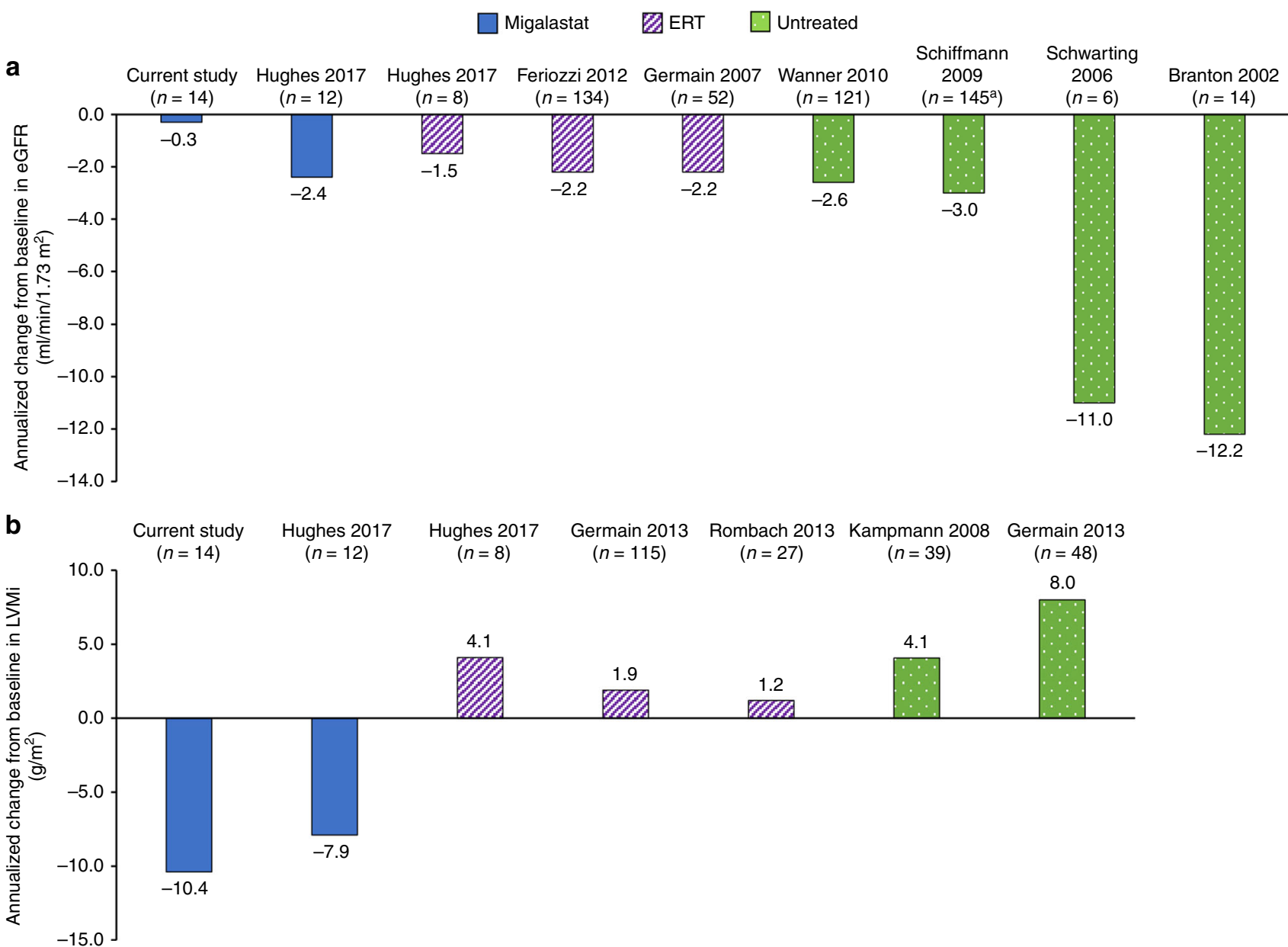

Fig. 2 Comparisons of published data for male patients with Fabry disease. Annualized changes in (a) renal function and (b) LVMi as reported in the literature for male patients who are either untreated or have been treated with migalastat or ERT. ${ }^{\text {a } C h a n g e ~ f r o m ~ b a s e l i n e ~ c a l c u l a t e d ~ u s i n g ~ t h e ~ w e i g h t e d ~}$ average for end-stage renal disease subgroups ( $n=128$ males with non-end-stage renal disease and $n=17$ males with end-stage renal disease). eGFR estimated glomerular filtration rate, ERT enzyme replacement therapy, LVMi left ventricular mass index. 
Further investigations are needed to obtain more definitive understanding of the pathophysiology of cardiomyopathy in Fabry disease and the effects of migalastat on cardiac tissues.

Elevated plasma lyso- $\mathrm{Gb}_{3}$ levels have become recognized as a diagnostic indicator for Fabry disease, especially for classic Fabry patients. ${ }^{37}$ Consistent with previous reports, ${ }^{37,38}$ baseline plasma lyso- $\mathrm{Gb}_{3}$ levels were highly elevated in male patients with the classic phenotype in FACETS. Migalastat was associated with substantial reduction in lyso- $\mathrm{Gb}_{3}$ in this patient subgroup. Despite the reduction, plasma lyso- $\mathrm{Gb}_{3}$ levels remained elevated above the normal ranges after 24 months of treatment. ${ }^{38}$ It has been reported that lyso$\mathrm{Gb}_{3}$ levels decreased but did not normalize despite years of ERT in male patients with the classic phenotype. ${ }^{37}$

In our study, the classic phenotype was defined as males with multiorgan system involvement and residual PBMC $\alpha$ Gal A activity $<3 \%$ of normal (Table 1 ). Of note, one of the GLA variants associated with the classic phenotype, p. Arg301Gln (p.R301Q), was originally identified in a Japanese male and characterized as a cardiac variant, a milder atypical variant with late-onset cardiac manifestations limited to the heart. ${ }^{39}$ Since then, at least one case of a more severe phenotype has been associated with this variant, including a 45-year-old male patient who had end-stage renal disease in addition to cardiomyopathy; this patient benefited from double heart and kidney transplant. ${ }^{40}$ The patient with the p. Arg301Gln variant in our study had multiorgan disease (stage 3 chronic kidney disease [eGFR $56 \mathrm{~mL} / \mathrm{min} / 1.73 \mathrm{~m}^{2}$ ], abnormal LVMi $\left[137 \mathrm{~g} / \mathrm{m}^{2}\right]$ ) and PBMC $\alpha-G a l$ A activity $1.9 \%$ of WT, thus fulfilling the criteria for inclusion in the classic phenotype subgroup.

Our study includes a descriptive analysis of the baseline disease characteristics and clinical outcomes of two patient subgroups in FACETS; the other patients subgroup consists predominantly, though not exclusively, of females. One potential limitation of our study is that outcomes based on indirect comparisons across studies should be interpreted with caution because of potentially confounding factors, such as variations in study design, inclusion/exclusion criteria, baseline demographics, and disease severity. In addition, the results reported for males with the classic phenotype from the FACETS study are derived from post hoc analyses in a relatively small group of patients observed for up to 24 months, a relatively short study duration for long-term data, given the progressive nature of Fabry disease. Lastly, GSRS-D has not been validated specifically in Fabry disease, although it has demonstrated reliability, stability, and construct validity across a range of GI disorders. No GI symptom rating scale has been validated in Fabry disease. Despite these limitations, our data show beneficial effects in male patients with the classic phenotype, and provide support for the use of migalastat in all patients with amenable GLA variants regardless of disease severity.

In conclusion, migalastat, a first-in-class oral pharmacologic chaperone, has previously demonstrated clinical benefits in patients with Fabry disease and amenable GLA variants. ${ }^{20}$ The data reported here establish that migalastat benefited male patients with the classic phenotype. During the phase 3 FACETS trial, migalastat treatment increased endogenous $\alpha$ Gal A activity, stabilized eGFR, reduced LVMi, improved diarrhea symptoms, and reduced PTC GL-3 inclusions and plasma lyso- $\mathrm{Gb}_{3}$ in male patients with migalastat-amenable GLA variants leading to the classic phenotype of Fabry disease.

\section{SUPPLEMENTARY INFORMATION}

The online version of this article (https://doi.org/10.1038/s41436019-0451-z) contains supplementary material, which is available to authorized users.

\section{ACKNOWLEDGEMENTS}

The authors thank the patients and clinical investigators who participated in the AT1001-011 study. The authors acknowledge Iulia E. Jurca-Simina for her contribution to data acquisition. Funding for the studies was provided by Amicus Therapeutics. Medical writing/editing support was provided by Sally Mitchell, Lei Bai, and Dana Francis of ApotheCom (Yardley, PA) and was funded by Amicus Therapeutics.

\section{DISCLOSURE}

D.P.G. has received honoraria and research grants from Amicus, Sanofi Genzyme, and Shire. K.N. has served as an advisor for Amicus, Shire, and Sanofi Genzyme, has received research support from Amicus and Shire, and has received travel support from Sanofi Genzyme. R.G. has received honoraria from Amicus, BioMarin, Sanofi Genzyme, and Shire. D.G.B. has received research funding, serves as a consultant, and is on the speaker's bureau for Amicus and Sanofi Genzyme, and has received research funding from Shire. D.A.H. has served as a consultant for and received research funding and honoraria from Amicus, Shire, Sanofi Genzyme, Protalix, and Actelion. L.M.B. has served as a consultant for Protalix. R.B.C. has served as a consultant for Amicus and has received grants from the National Institutes of Health. N.S., J.P.C., E.B., and J.A.B. are employees of and hold stock in Amicus. At the time the work was done, C.V. was an employee and held stock in Amicus. J.C.J. declares no conflicts of interest.

Publisher's note: Springer Nature remains neutral with regard to jurisdictional claims in published maps and institutional affiliations.

\section{REFERENCES}

1. Germain DP. Fabry disease. Orphanet J Rare Dis. 2010;5:30

2. Mehta A, Beck M, Eyskens $F$, et al. Fabry disease: a review of current management strategies. QJM. 2010;103:641-659.

3. Amicus Therapeutics Inc. Galafold (migalastat) amenability table website http://www.galafoldamenabilitytable.com. Accessed 15 October 2018.

4. Benjamin ER, Della Valle MC, Wu $X$, et al. The validation of pharmacogenetics for the identification of Fabry patients to be treated with migalastat. Genet Med. 2017;19:430-438.

5. Echevarria L, Benistan K, Toussaint A, et al. X-chromosome inactivation in female patients with Fabry disease. Clin Genet. 2016;89:44-54. 
6. Arends M, Wanner C, Hughes D, et al. Characterization of classical and nonclassical Fabry disease: a multicenter study. J Am Soc Nephrol. 2017;28:1631-1641.

7. El Dib R, Gomaa H, Ortiz A, et al. Enzyme replacement therapy for Anderson-Fabry disease: a complementary overview of a Cochrane publication through a linear regression and a pooled analysis of proportions from cohort studies. PLOS ONE. 2017;12:e0173358.

8. Schiffmann R, Kopp JB, Austin HA 3rd, et al. Enzyme replacement therapy in Fabry disease: a randomized controlled trial. JAMA. 2001; 285:2743-2749.

9. Eng CM, Guffon N, Wilcox WR, et al. Safety and efficacy of recombinant human alpha-galactosidase $A$ replacement therapy in Fabry's disease. $N$ Engl J Med. 2001;345:9-16.

10. Linthorst GE, Germain DP, Hollak CE, et al. Expert opinion on temporary treatment recommendations for Fabry disease during the shortage of enzyme replacement therapy (ERT). Mol Genet Metab. 2011;102:99-102.

11. Banikazemi M, Bultas J, Waldek S, et al. Agalsidase-beta therapy for advanced Fabry disease: a randomized trial. Ann Intern Med. 2007; 146:77-86.

12. Rombach SM, Smid BE, Bouwman MG, et al. Long term enzyme replacement therapy for Fabry disease: effectiveness on kidney, heart and brain. Orphanet J Rare Dis. 2013;8:47.

13. Weidemann F, Niemann M, Stork S, et al. Long-term outcome of enzyme-replacement therapy in advanced Fabry disease: evidence for disease progression towards serious complications. J Int Med. 2013; 274:331-341.

14. Lenders M, Stypmann J, Duning T, et al. Serum-mediated inhibition of enzyme replacement therapy in Fabry disease. J Am Soc Nephrol. 2016;27:256-264.

15. Galafold [summary of product characteristics]. Buckinghamshire, UK: Amicus Therapeutics UK Ltd; 2017.

16. Germain DP, Fan JQ. Pharmacological chaperone therapy by active-sitespecific chaperones in Fabry disease: in vitro and preclinical studies. Int J Clin Pharmacol Ther. 2009;47 suppl 1:S111-117.

17. Giugliani R, Waldek S, Germain DP, et al. A phase 2 study of migalastat hydrochloride in females with Fabry disease: selection of population, safety and pharmacodynamic effects. Mol Genet Metab. 2013;109:86-92.

18. Germain DP, Giugliani R, Hughes DA, et al. Safety and pharmacodynamic effects of a pharmacological chaperone on alpha-galactosidase A activity and globotriaosylceramide clearance in Fabry disease: report from two phase 2 clinical studies. Orphanet J Rare Dis. 2012;7:91.

19. Hughes DA, Nicholls K, Shankar SP, et al. Oral pharmacological chaperone migalastat compared with enzyme replacement therapy in Fabry disease: 18-month results from the randomised phase III ATTRACT study. J Med Genet. 2017;54:288-296.

20. Germain DP, Hughes DA, Nicholls K, et al. Treatment of Fabry's disease with the pharmacologic chaperone migalastat. N Engl J Med. 2016; 375:545-555.

21. Amicus Therapeutics announces approval of Galafold ${ }^{\oplus}$ (Migalastat) for Fabry disease in Japan [news release]. Cranbury, NJ: Amicus Therapeutics, Inc.; March 22, 2018. http://ir.amicusrx.com/news-releases/news-releasedetails/amicus-therapeutics-announces-approval-galafoldr-migalastat. Accessed 14 July 2018.

22. Migalastat [product monograph]. Buckinghamshire, UK: Amicus Therapeutics UK Ltd; 2017.

23. Migalastat [prescribing information]. Cranbury, NJ: Amicus Therapeutics Inc.; 2018.

24. Barisoni L, Jennette JC, Colvin R, et al. Novel quantitative method to evaluate globotriaosylceramide inclusions in renal peritubular capillaries by virtual microscopy in patients with fabry disease. Arch Pathol Lab Med. 2012;136:816-824

25. Sterneck M, Settmacher U, Ganten T, et al. Improvement in gastrointestinal and health-related quality of life outcomes after conversion from mycophenolate mofetil to enteric-coated mycophenolate sodium in liver transplant recipients. Transplant Proc. 2014;46:234-240.
26. Manger B, Hiepe $F$, Schneider $M$, et al. Impact of switching from mycophenolate mofetil to enteric-coated mycophenolate sodium on gastrointestinal side effects in patients with autoimmune disease: a phase III, open-label, single-arm, multicenter study. Clin Exp Gastroenterol. 2015;8:205-213.

27. Hwu WL, Chien YH, Lee NC, et al. Newborn screening for Fabry disease in Taiwan reveals a high incidence of the later-onset GLA mutation c.936 +919G >A (IVS4+919G>A). Human Mut. 2009;30:1397-1405.

28. Biegstraaten $M$, Arngrimsson R, Barbey $F$, et al. Recommendations for initiation and cessation of enzyme replacement therapy in patients with Fabry disease: the European Fabry Working Group consensus document. Orphanet J Rare Dis. 2015;10:36.

29. Schiffmann R, Warnock DG, Banikazemi M, et al. Fabry disease: progression of nephropathy, and prevalence of cardiac and cerebrovascular events before enzyme replacement therapy. Nephrol Dial Transplant. 2009; 24:2102-2111.

30. Schwarting A, Dehout F, Feriozzi S, et al. Enzyme replacement therapy and renal function in 201 patients with Fabry disease. Clin Nephrol. 2006;66:77-84.

31. Wanner C, Oliveira JP, Ortiz A, et al. Prognostic indicators of renal disease progression in adults with Fabry disease: natural history data from the Fabry Registry. Clin J Am Soc Nephrol. 2010;5:2220-2228.

32. Branton MH, Schiffmann R, Sabnis SG, et al. Natural history of Fabry renal disease: influence of alpha-galactosidase A activity and genetic mutations on clinical course. Medicine (Baltimore). 2002;81:122-138.

33. Germain DP, Weidemann F, Abiose A, et al. Analysis of left ventricular mass in untreated men and in men treated with agalsidase-beta: data from the Fabry Registry. Genet Med. 2013;15:958-965.

34. Kampmann C, Linhart A, Baehner F, et al. Onset and progression of the Anderson-Fabry disease related cardiomyopathy. Int J Cardiol. 2008; 130:367-373.

35. Feriozzi S, Torras J, Cybulla M, et al. The effectiveness of long-term agalsidase alfa therapy in the treatment of Fabry nephropathy. Clin J Am Soc Nephrol. 2012;7:60-69.

36. Germain DP, Waldek S, Banikazemi M, et al. Sustained, long-term renal stabilization after 54 months of agalsidase beta therapy in patients with Fabry disease. J Am Soc Nephrol. 2007;18:1547-1557.

37. Sakuraba H, Togawa T, Tsukimura T, et al. Plasma lyso-Gb3: a biomarker for monitoring Fabry patients during enzyme replacement therapy. Clin Exp Nephrol. 2018;22:843-849.

38. Boutin M, Auray-Blais C. Multiplex tandem mass spectrometry analysis of novel plasma lyso-Gb(3)-related analogues in Fabry disease. Anal Chem. 2014;86:3476-3483.

39. Kase $R$, Bierfreund $U$, Klein $A$, et al. Characterization of two alphagalactosidase mutants (Q279E and R301Q) found in an atypical variant of Fabry disease. Biochim Biophys Acta. 2000;1501:227-235.

40. Germain DP. A new phenotype of Fabry disease with intermediate severity between the classical form and the cardiac variant. Contrib Nephrol. 2001;136:234-40.

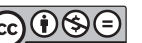

Open Access This article is licensed under a Creative Commons Attribution-NonCommercial-NoDerivatives 4.0 International License, which permits any non-commercial use, sharing, distribution and reproduction in any medium or format, as long as you give appropriate credit to the original author(s) and the source, and provide a link to the Creative Commons license. You do not have permission under this license to share adapted material derived from this article or parts of it. The images or other third party material in this article are included in the article's Creative Commons license, unless indicated otherwise in a credit line to the material. If material is not included in the article's Creative Commons license and your intended use is not permitted by statutory regulation or exceeds the permitted use, you will need to obtain permission directly from the copyright holder. To view a copy of this license, visit http://creativecommons.org/licenses/by-nc-nd/4.0/.

(C) The Author(s) 2019 